\title{
Positive Touch and massage in the neonatal unit: a British approach
}

\section{Cherry Bond}

Winnicott Baby Unit, St Mary's NHS Trust, London , UK

Semin Neonatol 2002; 7: 477-486

There is now a general trend towards a more baby friendly, family centred approach in the Neonatal Unit. Aspects of that approach - including positive touch and massage - are gaining in popularity. This has caused much debate due to the ambiguity surrounding the implementation and validity of the interventions, Here the impact of these complementary practices (not to be confused with complementary therapies) is discussed A review of the author's approach and potential guidelines for implementation is provided.

Key words: Positive Touch, massage, family, developmental care, preterm, infant, facilitation, NICU

\section{Introduction}

Staff working in Neonatal Intensive Care Units are increasingly looking for new ways to help newborn infants and their parents to endure the stressful experience of the modern day Neonatal Intensive Care Unit (NICU). Neonates not only deserve to receive the best biomedical-technological care, but also the best possible humane, psychological, and emotional support [1]. One way of helping to achieve this is with the use of a positive touch (PT) approach.

\section{What is Positive Touch}

Positive Touch involves various types of infant touch-interaction including handling, holding, kangaroo care and massage.

Positive touch is a term coined by the author and developed from her original booklet A Silent Dialogue, which was purely based on massage. The technique originated from Dr Frederick Leboyer's book Loving Hands which illustrates Shantala, a young Indian mother, massaging her baby in the streets of Calcutta [2]. Following qualification with 'The International Association of Infant Massage', and an introduction to a NIDCAP based programme, the approach was modified and re-named Positive Touch. This term reflects the adapted style that is now linked to a family centred developmentally sensitive philosophy so that it can be utilized for the smallest of infants in the NICU. The goal of PT is:

- To gently guide parents to some sense of mastery and ownership of their infant [3], in a difficult atmosphere of uncertain circumstances [4].

- To facilitate parental attunement to the behaviour of their infant, with the intention that because the brain develops in sequence, with more primitive structures stabilizing their connections first, early infant interaction is potentially beneficial to future development [5]. 
- To enhance the immature infant's experience. In the harsh NICU environment [61, avoiding prolonged stress, tactile aversion/avoidance and acute distress could have long-term health and behavioural benefits [7].

- To generally improve developmental outcomes on the premise of enhanced social, environmental and socio-environmental factors, which are known to be important predictors of good developmental and behavioural outcomes in preterm infants [8].

\section{The baby's experience}

The skin is the largest sensory organ of the body (about 2500 square centimetres in the newborn), and the tactile system is the earliest sensory system to become functional, the medium by which the infant's external world is perceived (9]. In premature birth the infant is called upon to make many adaptive responses to its new environment and, instead of building on the threads of sensory familiarity of touch, taste, smell and sound known in prenatal life, the transition to extrauterine life is a jarring disruption and a relentless introduction to a clinical world [101. Steven Hoath describes the skin as 'smart-material, which is a flexible and adaptive interface'. The preterm infant skin subserves multiple roles including: (1) a sensory surface for the infant; (2) a protective mantle; (3) a psychological/perceptual interface with caregivers and parents; and (4) an information rich surface for non-invasive monitoring [I I]. The possible effect of giving an infant comforting skin messages is therefore evident on many levels [121. Only a few decades ago it was believed that young children loved their parents simply because they provided nutrition, shelter and warmth, There was no evidence then, unlike now, that the brain contained emotional systems to directly mediate social bonds and social feelings [13]. This early behaviourist view began to change when it was shown that babies failed to thrive if they did not also receive both physical as well as emotional affection. The classic studies of Rene Spitz in the 1940s [141 demonstrated that babies in orphanages who did not have caring human contact often died prematurely. This lesson is being learned once more in Romania and former eastern block countries where infants from orphanages suffering from touch deprivation, achieve only half the normal height for their age.

The PT approach provides a caring sensory dialogue taking into account the sensitivity of premature skin and the consequence of touch on a fragile neonate, even when he is too unstable to be held. It gives the infant a sense of a comforting holding environment by a consistent caregiver (usually the parent).

\section{Who should do it?}

It is primarily practised by the parents who are the most appropriate givers of loving touch as they are the most consistent observers of their infant and have the essential emotional investment needed to give loving care. Consistent care giving is a goal that is rarely achieved in the neonatal nursery, yet it is a requirement and a probability for most newborn infants. Bender's work highlights the lack of constancy of all sensory experiences, including touch; he has looked at the innumerable caregiver's styles of handling, suggesting this may delay the baby's capacity to build up a consistent picture of his or her environment [15].

\section{When would you do it?}

Positive Touch is a way of counterbalancing the many and sometimes inevitable, unpleasant 
experiences, which seem to be a result of highly technical neonatal care. The approach works best if incorporated into the standard accepted care of the neonate. It would be as appropriate to give containment holding when performing a clinical procedure, such as insertion of an intravenous line, as when an infant is simply crying alone in the crib/incubator. In contrast, especially arousing types of touch such as massage could be done at a specific time when the baby indicates they are ready and the parent is present.

\section{Implementing Positive Touch}

The dialogue of touch is like any language: one needs to be able to listen and respond, and to both give and be receptive. Positive touch is always done 'with, not done 'to' a baby. The baby's responses/cues are pointed out in a way that shows that the baby is doing the teaching.

\section{Step 1. Preparation and observation}

When working sensitively with parents and infants at a time when they are both in a distressed state, it is necessary to just be with them without an agenda. This period offers an opportunity to simply listen with no preconceived ideas or judgement. Creating a space for parents to express emotions and fears can relieve some of their own burdens. This, in due course, frees them to think more about, and 'see' their baby [16].

Parents (and other caregivers) can find it difficult to identify with the preterm infant, as their cues are masked in the subtle veil of prematurity $[17,181$. Pointing out individual infant cues/reactions that signal engagement, disengagement, and selfregulatory abilities, can guide the parent towards appropriate, safe touch for their baby at any given time. This interactive 'language' can be learnt and used in any situation so that care is always safely adapted to the individual infant's tolerance levels [19]. Parents who become experienced with this 'dialogue' can then be continually aware of their baby's amazing capabilities, and move forward in understanding the developmental possibilities, strengths and vulnerabilities of their baby, so that a whole picture of their unique child emerges rather than merely his medical condition.

\section{Step 2. Parents in attendance, without touch}

Extremely premature infants, those who are recovering from surgery, or infants who are very sick and/or sedated, may not tolerate even the slightest of touches. In this situation, parents can be encouraged to sit by the bedside and quietly make their presence fell by leaning close and putting their hand(s) a few centimetres away from their baby's head or/and feet. Facilitating the parents to take slower, deeper breaths themselves can help ease tensions. If they are able to; picturing a soothing colour that surrounds the infant can also help relax the parent, which in turn can have a soothing impact on the baby. Although there is no direct contact, the parents can experience a connection with their infant, which can provide an optimum opportunity for involvement at a time of great stress and uncertain survival [20]. The nature of a neonatal loss is often sudden and unexpected; it is vital to encourage some kind of contact during these early bedside encounters. It demonstrates from the outset that we always value the baby as being part of a family.

\section{Step 3. Initiating touch (permission)}

Ideally every touch we give should begin by preparing the baby with a thought or a word of 
intention of the impending approach (we infant massage instructors call this 'permission').

The decision to initiate touch, or not, is judged by taking note of the baby's behavioural state, medical condition, and watching for signals of acceptance. This crucial step demonstrates that we respect the baby's ability to be participants in their own caregiving. Preparing the infant for impending touch also promotes trust and supports the child's emerging sense of self and ultimate behavioural competency [21]. Enduring continual approaches that cause sudden, painful or startling responses, may lead to hypo-responsive infant behaviours that are commonly seen in graduates of the NICU, and are possibly a further factor influencing behavioural outcomes [22].

To promote infant readiness the environment may also need to be adjusted, e.g. reduce lighting, cut down noise levels, and ensure warmth and comfort.

\section{Step 4. Still holding/containment}

Still or containment/holding can be a way of providing stability and predictability for the NICU infant, and also enables parents to gain confidence, especially in the early days, or whenever their baby may be medically unstable [23].

Still touch/holding progresses with a slow approach, resting a hand(s) on the baby [24], with the effect of heaviness in the touch. This technique is also useful for infants who are already very fretful, or recovering from surgery. The initial inherent touching instinct of parents involves light fingertip stroking [25], which is generally not tolerated by fragile infants (26). Anxious parents may need a reassuring hand on top of theirs to steady their first tentative touch. Containment holds that suit the baby can also be used during procedures when the baby needs support [27].

Containment support is performed in a sequence as in step two, but with direct contact, the caregivers hands being cupped around the infant's head, possibly feet or hands, depending on the individual infant and his reactions. The positive touch instructor can work with the parents to help them discover the best way to connect to, relax and soothe their baby. Linda Harrison, a neonatal nurse from the University of Alabama, has evaluated these modes of gentle human touch and suggests that they are generally safe for young preterm infants, but emphasizes that individual infant responses to touch need to be continuously monitored by NICU staff and parents [28].

\section{Step 5. Pacing}

It is important to adjust the pace of any touch given to each individual infant. Any touch may elicit an initial 'avoidance response', as most NICU infants are hypersensitive. The more attractive to the stimulus (such as touch, the human voice and face), the more the infant will overreact [29]. To help an infant respond to interaction, such as touch/massage, and instigate self-regulabon, each stimulus should be adjusted in its speed, intensity, and duration. Often a premature or stressed infant can only take in, and respond to, one modality at a time. Caregivers who respect and respond to the infant's threshold for stimuli, will maintain infant stability and broaden the infant's capacity for interaction.

\section{Step 6. Kangaroo care}

When looking back 8000 years, the evidence is that our ancestors always carried their babies close to them. It is believed that baby-carrying devices, made from animal skin, may have been the earliest form of human appliance. Culturally there are many different carrying styles that suit a 
variety of life-styles and traditions [30].

In 1978, in response to the overcrowding of the Neonatal Unit at the largest public obstetrics facility, the Mother \& Child Institute of Bogot $\sim$, Dr Edgar Rey-Sanabria, a paediatrician and professor A the National University of Colombia, developed the so-called kangaroo mother technique. A programme was commenced in 1979, by Drs Martinez and Rey-Sanbria (Kangaroo Foundation) [31] which resulted in an amazing increase in the number of babies surviving - 72\% under I kg and 89\% between 1-1.5 kg. There was also an improvement in the higher weight range and the number of mothers who abandoned their babies fell from 34 in one year to 10 [32].

Kangaroo care (KC - sometimes named skin-toskin holding) has gradually been adopted by the economically affluent, industrialized countries, not in an attempt to save lives, but in an attempt to re-humanize care of the baby and parent in a response to the trend for uncompromizing, dehumanizing highly technical neonatal treatment [33]. Early tactile contact was seen to make a difference in mothers accepting the reality of a preterm birth [34]. Even very small babies in highly sophisticated NICU's [35] can benefit from being held in the 'kangaroo-type' position [36]. Feldman and Eidelman have systematically analysed various modes of intervention therapy and $\mathrm{KC}$ is suggested as the intervention that most logically meshes the premature infant's need to develop state regulation while facilitating sequential sensory development and promoting mother-infant attachment [37].

Although named 'skin to skin' it is not necessary to undress a baby for kangaroo care, as the process of removing and then re-dressing [he infant may be very disruptive, especially when they are asleep when put back into their bed. Every neonate will have a different receptivity, tolerance and energy level. Taking a small/ventilated baby out of an incubator needs planning, with consideration of the baby's individual needs, daily rhythms of sleep and the willingness of the family [38].

The significance of skin to skin, KC, holding, cuddling and close loving touch has yet to be quantified, but it must- be considered, given the inherent need for parents to carry out this kind of care, as to whether we can ignore the humane rights of the family to provide treatment such as this [39).

\section{Step 7. Letting go}

Departure of touch begins slowly, as the initial approach, with a still resting hand. Before letting go, intention of the impending departure is transmitted verbally or with silent intent. If the baby is hypersensitive and reacts distressingly to the departure, [he letting go process can be restarted and completed more slowly; adjustments in the infant's position, bedding or environment may also help to settle him. This can assist the infant to maintain a quiet sleep state and maintain stability after an intervention of any kind.

Support at difficult times

Providing hands-on-containment and support, at times when an infant's stability is being challenged, e.g. examinations and procedures (including physical checks, scans, X-rays, and eye examinations), will support the infant's comfort and well being. Support that is preplanned and adjusted to the individual infant can help the baby regulate their physiologic, motoric, behavioural and state systems, all of which can aid the clinician as well as the infant. 
Examples of support:

- Containing the infant's extremities in a flexed position.

- Offering opportunities for grasping onto a finger, a cloth or bedding.

- Giving rest periods (pacing) during the stressful procedure.

- Offering a pacifier or other sucking opportunities.

Maintaining the support after an aversive intervention can re-stabilize the infant, making these difficult times a strengthening accomplishment of competence, rather than a further weakening and depleting event.

\section{Adapting care-times}

Any care-giving occasion is an opportunity to adjust handling in a positive style [40]. Positive experiences for the infant can be integrated into all routine nursing policies and procedures [411. Focus on the parents' needs and strengths is also part of the approach. Parents who share in infant-care have been shown to have a greater sense of self-esteem and attachment behaviours [42]. Examples of adaptive care times:

- Bath times are an ideal opportunity for parents to handle and observe their baby while the infant deals with the numerous challenging stimuli a bath presents [43]. The infant's first bath is an important parenting undertaking. If relaxed and unhurried, the bathing process can become a time to familiarize the infant to handling which includes parent-infant interaction and fulfilment, as well as for hygiene and efficiency.

- Mouth care can incorporate massage techniques to promote infant feeding skills. In a study of preterm infants born between 26-29 weeks gestation, an early or oral stimulation programme accelerated the transition to full oral feedings [44]

- Changing temperature or oxygen probes can be PT adapted by incorporating some still holding or simple relaxing strokes with oil, to avoid infant distress and skin trauma [45]

- Inserting a feeding tube can be adapted using PT techniques so that it is achieved without causing distress.

\section{Progress to massage}

Massage is one of the oldest forms of treatment, and a routine part of infant care throughout the world. Infant massage is motivated by various cultural and familial foundations, some to do with health and protection frcim the elements, others with emotional well being, and still others with beauty [46]. In the NICU, the majority of touch infants are experiencing is mostly associated with medical, technical and nursing procedures which may not be positive experiences for the infant [47]. Most new parents express the desire to touch and stroke their infants and therefore neonatal staff are increasingly looking for interventions, such as massage, to address this need (Fig 1).

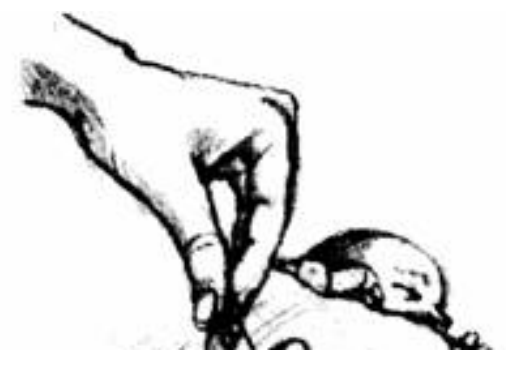

Figure 1. Positive parental touch can be a powerful, nuturing dialogue. 
The word massage (Oxford Dictionary) infers rubbing or kneading the skin with the hands to stimulate or manipulate. No wonder it can cause concern if referred to in conjunction with fragile neonates! Although massage is a more active exchange than the PT sequence, and can be stimulating [481, it can also be soothing and relaxing [491. Any stimulation is better when performed in a developmentally sensitive manner, and massage is no exception to this rule. Massage strokes should only be initiated when the baby shows signs of being able to tolerate positive still touch, i.e. without displaying behavioural and physiological instability [50].

Parents will often stroke and massage instinctively, but the way a baby is moved, and protected from becoming disorganized, is an important part of teaching massage and vital for safety. In the Cochrane review on preterm massage, Andrew Vickers states, 'where massage is currently provided by nurses, consideration should be given as to whether this is a cost-effective use of time' [51]. In a preterm massage study looking at enhanced weight gain [52] mothers who were given guidance, were able to achieve the same positive effect as that of trained professionals.

Babies in specialized units are coping with many taxing demands such as medical interventions, examinations, and developmental challenges like growing and feeding, therefore, massage prescribed at predetermined times and of a set duration and format, should be avoided. It is better to offer some form of positive skin stimuli regularly (at least each day if possible), and in a predictable way (by parents) so that the infant experiences a consistent balance of positive versus negative touch.

Movement begins on the part of the body where the baby seems to like still touch (often the head, hands or feet), with one slow but firm movement at a time. A deeper pressure, than that of a tickle stimulus, has been shown to produce enhanced behaviour in response to massage movements [53]. Rhythmical massage movements continue only if the baby remains organized; the parents are encouraged to watch and be ready to change their handling responses as the baby changes. The baby's trust and anticipation -tolerance for massage builds slowly over a number of sessions.

\section{Massage groups in the community}

When a baby goes home, massage groups can provide the parents with an opportunity to meet other families, share ideas, gain support and appreciate that their baby can fit-in-with and 'hold their own' with infants who were born at term [54].

\section{Teaching and demonstrating}

\section{Infant massage training}

To ensure that touch therapies are safe and appropriaEe in the NICU, sound knowledge of the principles of developmental care is essential. Neonatal staff, wanting to instruct patients in infant massage, should then seek appropriate [raining. The ideal massage course is advisably one taught by specific infant massage trainers (IAIM) [55] who are aware of infant cues and have international links to NICU massage advisors who have worked with preferm infants themselves.

When introducing massage into an NICU there is a need for guidelines to be set so that standards can be maintained and the risk to the baby and family are minimized [56]. 


\section{Dolls}

Using a doll for all demonstration purposes proves to be a simple yet powerful way of enabling parents to find their own unique way of building a consistent relationship, through touch, with their baby. Special soft-bodied premature dolls are available which are designed as a massage tool [57].

\section{Massage oils}

Massage with oil ensures a smooth, gliding surface without friction and therefore touch is better accepted ]581. When choosing oil for use in an NICU there are several con'siderations:

1. A plant based oil that is easily absorbed into the epidermis, is better for the skin than a mineral based oil which can leave a sticky, skinoccluding film on the skin's surface.

2. Check with a paediatric allergist and the local anaphylactic centre to ensure you use low allergen-risk oil. This may differ depending on the country.

3. Refined or purified oil is more likely to be free from any unwanted additives such as yeasts and moulds which can be present in some cold pressed oils. The refining process should also remove any allergens [591 and the shelf life will be considerably extended.

4. It is important to use odour-free oil in the NICU, as most added scents are either chemical or essential oil based.

5. Individual bottles (less than $50 \mathrm{ml}$ ) avoid cross contamination; the small quantity also ensures that the oil is used quickly which guards against it oxidizing.

Examples of oil that fit the above criteria are refined sunflower or fractionated coconut. Topical use of linoleate-enriched oils may enhance skin barrier function and improve outcome in neonates with compromised barrier function [60].

\section{The parents experience}

Every parent, regardless of personal philosophy, and every infant regardless of birth history or disposition, should have the opportunity to experience the lifelong benefits of early attachments that are loving, healthy and secure [61].

There have been numerous reports on the effects of parental stress and family adjustment in the NICU [62-641: it is clearly difficult and traumatic time for most families.

Parents may not know what to do with the fear and fatigue that is created as a result of having a child in the NICU. It requires a good holding environment in order to be well tolerated and well dealt with. The PT instructor can assist the process of adjustment by giving parents opportunities for rewarding activities such as touching and holding experiences.

The majority of attachment research has focused on the mother, but in the NICU the closeness of the baby may be more equally shared between both parents, than it would be in other normal 
settings. It is often the father who has the earliest contact with the baby on the NICU, and the earlier fathers hold their babies; the quicker the attachment [651. Fathers who are highly involved with their infants have been found to be as tactile-sensitive as were their mothers [66]. Allan Schore's work details the neurobiology of attachment, his observations show fathers who regularly interact with their infant, can develop greater neural connections in the right brain area [67]. Preterm babies are known to draw fathers into deeper levels of involvement than comparably healthy babies [68] and fathers who visit frequently are also more connected to their babies in later life [69]. Fathers can also benefit from early contact with their infant. In a study in Israel, KC had a significant positive impact on the infant's perceptual-cognitive and motor development and on the parenting process at 6 months of age [70].

\section{Staff}

Providing a positive touch approach and highlighting parental importance can enhance the nurse's role as caregiver. The challenge for staff is in creating a holding environment that assists the progress of parental empowerment, and allows the family to become partners in their baby's care. Staff would also benefit from a holding environment, and can accumulate a range of self-protective mechanisms for dealing with the high stress and uncertainty, which is the every day norm in the

NICU [711. Dealing with parents as well as the high infant workload, can be extremely stressful especially when staff feel unprepared and at times unsupported themselves [72]. Providing massage treatments for the staff themselves [73] and parents [741 is a way to promote an understanding of the impact of touch while having the spin-off of keeping the staff healthier, happier and feeling valued [751.

\section{Conclusion}

The preterm delivery constitutes a physical [76] and emotional crisis, which can have significant and prolonged family repercussions [77]. There is the potential that acute and prolonged distress can go unrecognized and unrelieved.

Positive Touch and massage is proposed as a means of reducing the negative impact on the neonate and his family. There is now ample neuroscientific evidence that during development, behaviour acts in concert with the environment, to establish structural changes in the brain that last a lifetime [78]. The consequences of early nurturing touch may therefore favourably influence experience dependent brain maturation [791, the organization of infant stress responses [80], the tactile system and self-regulation [811, all of which could have beneficial effects on later behaviour.

Touch is a form of communication that transcends almost all culture [82]; it is a regulator, and organiser [831 and a source of information (stimulation as well as deprivation), and may be integrated into a therapy [841. Positive Touch aims to optimize care to make it more gentle and humane in the present, and less damaging in terms of outcome, by fostering a dialogue between the baby and family. The touch is adapted to the individual, acknowledging the infant's strengths and vulnerabilities with respect for parental involvement as a prerequisite.

This approach to infant care is also designed for, and in admiration of staff in Obstetric and Neonatal Intensive Care Units, who tend to survive the best they can, and may benefit from support from a multidisciplinary team [851 including a Positive Touch specialist. It aims to help 
them deal better with the strain, which is put upon them, so that they will increase their job satisfaction [86] and allow them to offer more assistance to both infants and parents.

By promoting holistic nursing care, and while offering an opportunity to accept the parents' contribution as an essential part of the baby's development, we can mediate the trauma of intensive care and its distressing effects. Offering care that is both life-saving in essence and also enriched by loving touch and empathy, embodies the very heart of true nursing care.

References

I Leven A, Humane neonatal care initiative. Acta Paediat 1999; 88: 353-355. rica

2 Leboyer F. Loving Hands: the traditional Indian art of baby massage. (Original copy by Collins 1977), Newmarket Press, New York , 1997.

3 Likierman M. Maternal love and Positive projective identification. Journal Of Child Psychotherapy 1988; 14(2): 29-46.

4 Mincle K, Whifelaw A, Brown J, Fitzhardinge P. Effects of neonatal complications in premature infants on early parent-infant interactions. Developmental AIedicine and Child Neurobiology 1983; 25: 763-777.

5 Nash JM. Fertile minds. Time Allagazine 1997, 149(5)www.time.com/lime/ agazine/1997/dom/970203/covero.' himi.

6 Barker DP, Rutter N - Exposure to invasive procedures in neonatal intensive care unit admissions. Archives of Disease 1995; 72(1): 47-48 ,

7 Panksepp J. Loneliness and the social bond. In: Affective Neuroscience: the foundations of human and animal emotions. Oxford University Press 1998; 14: 261-299.

8 Wolke D ' Psychological development of prematurely born children. Archives of Disease in Childhood 1998; 78: 567-570.

9 Montague A. Touching, the human significance of the skin. Harper \& Row 1986; 3-46.

10 Hardy H. The experience of early infancy for the prelerm infant. In: Tracy N. (ed .) Parents of Premature Infants: their Emotional World. Whurr Publishers, London and Philadelphia , 2000; 14115: 195-212.

11 Hoath SB. The skin as a neurodevelopmental interface. In: The Physical and Developmental Environment of the High-risk Infant. Conference Syllabus 2001, 39-40.

12 Hoalh S, Visscher M, Heaton C, Neale H. Skin science and the future of dermatology. Journal of Cutaneous Medicine and Surgery 1998- 3: 2-8. 
13 Panksepp J, Nelson E, Bekiedal M. Brain systems for the meditation of social separa lion-dis tress and social-reward: evolutionary antecedents and neuropeptide intermediaries. Annals of the New York Academy of Science 1997; 807: 87-100.

14 Spitz R. Hospitalism. The Psy h 1945; 1: 53-74.I naylic Study of the Child

5 Bender H. Neonatal intensive care. In- Davis Fallowfield L. (eds) Counselling and Communication in Health Care. Chichester : John Wiley \& Son, 1990.

16 Eriksson BS, Pehrsson G. Evaluation of ' scho-social support to parents with an infant bom preleprmy. Journal of Child Health 2002; 6(1): 19-33.

17 Johnson CC, Stephens B, Craig KD, Grunau RV. Developmental changes in pain expression in premature, fullterm, and two- and four-month-old infants. Pain 1993;

52(2): 201-208.

18. Mouradian LE, Als H, Coster WJ. Neurobehioral functioning of healthy p,elerm infants of varying gestational ages. Journal Of Developmental and Behavioral 21(6): 408-416. Pediatrics 2000;

19 Brown JV, VandenBerg MA. Considerations for touch and massage in the neonatal intensive care unit. Neonatal

Network 19(l). 61-64.

20 VandenBerg KA. Supporting parents in the NICU: guidelines for promoting parent confidence and competence. Neonatal Network 2001; 19(8)- 63

21 Stem DN. Mothers' - -64. emotional needs. In: New Perspectives in Early Emotional Development. Johnson

Pediatric Institute Ll \& Johnson 1998: 65-91. d., Pediatric Round Table Series USA ,

22 Case-Smith J, Butcher L, Reed D. Parent's report of sensory responsiveness and temperant in prelerm infants.

American Journal of Occupational Therapy 547-555. 1998; 52:

23 Harrison L, Olivet 1, Cunningham K, Bodin MB, Hicks C. Effects of gentle human [Ouch on preterm infants.

Neonatal Network 1996; 15: 35-42. handbook for loving parents, 3rd edn. Souvenir Pr

24 McClure V. Your premature baby. In: Infant A allagl: a 11: 157-165. ess, 2001;

25 Klaus MH, K,nnell N, Plumb N, Zuehlke S. Human maternal behavior a, the first contact with her young. Pediatric, 1970; 45: 197-205. 
26 Schanbe,g S. The genetic basis for [ouch effects. In: Field T. (ed.) Touch in Early Development. Lawrence Eflbaum A,,oci_ales, Mahwah , New Jersey . Hove , UK , 1995; 6: 67-79.

27 Corff KE. An effective comfort measure for minor pain and stress in preferm infants: facilitating tucking. Neonatal

Network 1993; 12(8): 74.

28 Harrison LL, Williams AK, Berbaum ML, Stem JT, Leper I Physiologic and behavioral effects of gentle human touch

on preterm infants. Research in Nursing and Health 2000; 23(6): 435-446.

29 Brazellon TB.. Touchpoints: Your Child's Emotional and Behavioral Development. Perseus 350-35 . Books, 1992; 32:

30 Mannoni C. Role of bodily Practices with newborn in the p1reavcelilcoepsmiern [ Africa and India . Archives de Pediatri, 2001; 8(8): 882-888. of cultural identity. Examples of bodily

31 Kangaroo Foundation. Santaf6 de Bogo[6, DC Colombia, Transv 39A No. 46-29. Tel/Fax: 22270 : E-mail: herchar5@colomsal.net.co; Webpage: kangaroo.javeriana.edu.co

32 Whifelaw A, Sleath K. Myth of the marsupial mother: Home care of very low birth weight babies in Bogota ,

Colombia . Lancet 1985; : 1206-1208.

33 Charpak N, Ruiz JG, Figueroa Z. Humanizing neonatal care. Acta Pediatrica 2000; 89: 501-502.

34 Reid T. Maternal identity in prelerm birth. Journal of Child Health Care 2000; 4(l): 23-29.

35 Anderson GC, Dombrowski MAS, Swinth JY. Kangaroo care: not just for stable preemies anymore. Reflections on

Nursing Leadership 2001; 27(2): 32-34, 45 .

36 Ludington-Hoe SM, Swinth JY. Developmental aspects of kangaroo care. Journal of Obstetric, Gynaecological and Neonatal Nursing 1996; 25(8): 691-703.

37 Feldman R, Eidelman Al. Intervention programs for premature infants. How do they effect development. Clinics in Perinatology 1998; 25(3): 613-626.

38 Gale G, VandenBerg KA. Kangaroo care. Neonatal Network 1998; 17(5): 69-71.

39 Charpack N, Figueroa de Calurne Z, Ruiz JG. The Bogota declaration on kangaroo mother care: conclusions at the second international workshop on the method. Acta Paediatrica 2000; 89: $1137-1140$. 
40 Buehler D, Als H, Duffy FH, McAnulty GB, Leiderman J. Effectiveness of individualized developmental care for low-risk preterm. infants: behavioral and electrophysiologic evidence. Pediatrics 1995; 96(5): 923-932.

41 Warren 1. Difficult procedures and daily care. In: Guidelines for Infant Development in the Newborn Nursery, 3rd edn, Winnicott Baby Unit, St Mary's Hospital, London, 2001; 3: 69-92.

42 Chen CW, Conrad B. The relationship between maternal self-esteem and maternal attachment in mothers of hospitalised premature infants. Journal of Nursing Research 2001; 9(4): 69-82.

43 Peters KL. Bathing premature infants: physiological and behavioural consequences. American Journal of Critical Care 1998; 7(2): 90-100.

44 Fucile S, Gisel E, Lau C. Oral stimulation accelerates the transition from tube to oral feeding in preterm infants. Journal of Pediatrics 2002; 141(2): 230-236.

45 Heath SB, Narendran V. Adhesives and emollients in the preterm infant. Seminars in Neonatology 2000; 5(4): 289-296.

46 Dunham C, Myers F, Barnden N, et al. Mamatoto: A Celebration of Birth. Virago Press , UK, 1991.

47 Horton J, Waldenstrom U, Bowman E. Touch of low birth weight babies in neonatal intensive care: observations over a 24 hour period. Journal of Neonatal Nursing 1998; 4(2): 24-28.

48 Scafidi F, Field T, Schanberg S, Bauer C, Tucci K, Roberts J, Morrow C, Kuhn C. Massage stimulates growth in preterm infants: a replication. Infant Behavior and Development 1990; 13: 167-188.

49 Wheeden A, Scafidi FA, Field T, Ironson G, Valdeon C, Bandstra E. Massage effects on cocaine-exposed preterm neonates. Journal of Developmental and Behavioural Pediatrics 1993; 14(15): 318-322.

50 Burns K, Cunningham N, White-Traut R, Silves[tri J, Nelson M. Infant stimulation: modification of an intervention based on physiologic and behavioural cues. JOGNN 1993;581-588.

51 Vickers A, Ohisson A, Lacy JB, Horsley A. Massage for promoting growth and development of preterm and/or low birth-weight infants (Cochrane Review). In: The Cochrane Library, issue 2, 2001. Oxford : Update software.

52 Ferber SG, Kuint J, Weller A, Feldman R, Dollberg S, Arbel E, Kohelet D. Massage therapy by mothers and trained professionals enhances weight gain in preterm infants. Early Human Development 2002; 67(1-2) 3-45.

53 Field T. Enhancing growth. In: Touch Therapy. Churchill Livingstone, 2000; 1: 4-11.

54 Adamson S. Teaching baby massage to new parents. Complimentary Therapies in Nursing and Midwifery 1996; 2: 151-159. 
55 The International Association of Infant Massage (IAIM). UK Office, 56 Sparsholt Road , Barking, Essex IG11 7YQ. Tel/Fax: 0208591 1399; E-mail: mail@iaim.org.uk; Website: www.iaim.org.uk

56 Wall A. Agreeing a standard for infant massage: not a soft touch. Journal of Neonatal Nursing 8 (3): 93-96.

57 Touch Needs. Suppliers of professional resources for positive touch teachers. Touch Needs, Unit 3, Elms Farm Industrial Park , Bramshall, Uttoxeter, Staffordshire ST14 5BE. Tel./Fax: 01889 560260. E-mail: enquiries@ touchneeds.com; Web: www.touchneeds.com

58 Field T, Schanberg S, Davalos M, Malphurs J. Massage with oil has more positive effects on normal infants. Pre and Perinatal Psychology journal 1996; 11(2): 75-80.

59 Hourihane J, Bedwani S, Dean T, Warner J. Randomised, double blind, crossover challenge study of allergenicity of peanut oils in subjects allergic to peanuts. British Medical journal 1997; 314: 1081-1087.

60 Darmstadt CL, Mao-Qiang M, Chi E., el al. Impact of topical oils on the skin barrier: possible implications for neonatal health in developing vountries. Acla Paediatrica 2002; 91(5): 546-554.

61 McClure V. The Tao of Motherhood. Nucleus Publications, USA , 1994.

62 Tracey N, Blake P, Warren B, Hardy H, Enfield S, Schein P. A mother's narrative of premature birth. Journal of Child Psychology 21(l): 43-64.

63 Doering LV, Moser DK, Dracup K. Correlates of anxiety, hostility, depression, and psychosocial adjustment in parents of NICU infants. Neonatal Network 2000; 19(5): 15-22.

64 Pinelli J. Effects of family coping and resources on family adjustment and parental stress in the acute phase of the NICU experience. Neonatal Network 2000; 19(6): 27-36.

65 Sullivan JR. Development of father-infant attachment in fathers of preterm infants. Neonatal Network 1999; 18(7): 33-39.

66 Kaitz M, Lapidot P, Brenner R, Eidelman AL. Parturient woman can recognise their infants by touch. Developmenfal Psychology 1992; 28: 35-39.

67 Schore AN. Effects of a secure attachment relationship on right brain development, affect regulation, and infant mental health. Infant Mental Health journal 2001; 22(1-2): 7-66.

68 Pleck J. Paternal Involvement, Levels, Sources, and Consequences. New York , NY : John Wiley \& Sons, 1997.

69 Levey-Shiff R, Hoffman MA, Mogilner S, Levinger S, Mogilner MB. Fathers' hospital visits to their preterm infants as a predictor of father-infant relationship and infant development. Pediatrics 1990; 86(2): 289-293.

70 Feldman R, Eidelman Al, Sirota L, Weller A. Comparison of skin-to-skin (kangaroo) and 
traditional care: parenting outcomes and preterm infant development. Pediatrics 2002; 110(l): $16-26$.

71 Gorski PA. Experience following premature birth: stresses and opportunities for infants, parents and professionals. In: Call JD, Galenson E, Tyson RL (eds) Frontiers of Infant Psychiatry, vol. 2. New York : Basic Books, 1998.

\section{Bond}

72 Fischer JE, Calame A, Dettling AC, Zeier H, Franconi S. Experience and endocrine stress responses in neonatal and pediatric critical care nurses and physicians. Critical Care Medicine 2000; 28(9): 3281-3288.

73 Field T, Quinfino 0, Hentelef T, Wells-Keif L, Delvecchio-Feinberg G. Job stress reduction therapies. Alternative Therapies in Health and Medicine 1997; 3(4): 54-56.

74 Field TM. Massage therapy effects. American Psychologist 1998; 53(12): 1270-1281.

75 Peebles-Kleiger MJ. Pediatric and neonatal intensive care hospitalisation as traumatic stressor: implications for intervention. Bulletin of the Menninger Clinic 2000; 64(2): 257-280.

76 Modi N. The role of early experience in infant development - enhancing outcome after extremely preterm birth. In: The Role of Early Experience in Infant Development. Johnson \& Johnson Pediatric Institute Ltd, USA , Pediatric Round Table Series, 1999: 267-282.

77 Wereszczack J, Miles MS, Holditch-Davis D. Maternal recall of the neonatal intensive care unit. Neonatal Network 1997; 16(4): 33-39.

78 Schore AN. Affect Regulation and the Origins of the Self., the Neurobiology of Emotional Development. Lawrence Erlbaurn Associates, 1999: 303-307

79 Schore AN. The experience-dependent maturation of an evaluative system in the cortex. In: Pibram KH (ed,)

Brain and Values: is a Biological Science of Values Possible. Proceedings of the Fifth Appalachian Conference on Behavioral Neurodynamics. Mahwah , NJ : Lawrence Erlbaurn Associates, 1998..

80 Anand KJS. Relationships between stress responses and clinical outcome in newborns, infants and children. Critical Care Medicine 1993; 21: 358-359.

81 Mathai S, Fernandez A, Mondkar J, Kanbur W. Effects of tactile-kinesthetic stimulation in preterms: a controlled trial. Indian Pediatrics 2001; 38(10): 1091-1098.

82 Montague A. Culture and contact, In: Touching: the Human Significance of the Skin. Harper and Row, New York., 1986; 8: 292-400.

83 Schore A, Attachment and the regulation of the right brain. Attachment and Human Development 2000; 2(1):: 23-47. 
84 Barlow J, Cullen L. Increasing touch between parents and children with disabilities: preliminary results from a new programme. Journal of Health Care 2002; 12(l): 7-9.

85 Shannon JD, Gorski PA. Health-care professionals' attitudes toward the current level and need for developmental services in the noonatal intensive care units, Journal of Perinatology 1994; 14 (6): 467-472.

86 Gorski PA. Caring relationships: an investment in health. Public Health Reports 2000; 115 (2-3): 144-150. 